Goldschmidt 2021 Abstract

https://doi.org/10.7185/gold2021.7775

\title{
Quantifying silicate weathering fluxes in rivers
}

MIKE BICKLE

University of Cambridge

Presenting Author:mb72@esc.cam.ac.uk

Distinction of the inputs into rivers from silicate minerals, carbonate minerals, and salts is critical to estimating the silicate weathering fluxes thought to act as a major feedback controlling the Earth's climate on long timescales. Distinction of the inputs is generally achieved by using estimates of their typical cation ratios (e.g. $\mathrm{Ca} / \mathrm{Na}$ ) to partition the riverine cation fluxes. This may be by estimating the source ratios from analysis of rocks or riverine suspended loads and using these in a forward calculation or by making a priori estimates of the cation ratios and solving the set of mass balance equations for both the end-member ratios and the fractions derived from the various inputs in an 'inverse calculation'. However as the water chemistry described by $m$ components derived from $n$ inputs must lie in an $R^{n}$ sub space of the $R^{m}$ chemical concentration hyperspace, it is only possible for the inversion program to locate the end-member compositions in the subspace but not locate their positions within the subspace, so that the magnitude of relative inputs remains undetermined. However for a system with $m$ components (e.g. cation concentrations or isotope ratios) from $n$ different inputs (eg. carbonates, silicates, salts), if $n-1$ element ratios are known for each input, then the other $m-n$ ratios may be determined from best fits of the water chemistry to the $R^{n}$ sub space. Since there is considerable uncertainty about the element-ratios that characterise weathering inputs it is suggested that a better approach is to use estimates of the best known element ratios to infer the less well known as a test of the approach. The assumption that rock or sediment element ratios characterise the inputs is particularly suspect given that weathering is generally the product of incongruent dissolution. Further with multiple components and inputs, uncertainty estimates can become very large. The sensitivity and limitations of the approaches are examined by modelling both synthetic and real data. 